\title{
Electronic Robustness by Circuit Polling and Reconfiguration
}

\author{
György Györök \\ Alba Regia University Center \\ Óbuda University \\ Budai Str. 45, H-8000 Székesfehérvár \\ gyorok.gyorgy@arek.uni-obuda.hu
}

\begin{abstract}
Nowadays the uses of electronic circuits, often the opposite trend are seen. To produce a hand are getting cheaper circuits, on the other hand becoming more reliable ones. The first distinction is certainly true in electronic circuits which are used in commercial application, even just thinking of cheap consumer electronics [1] [18] [13] [12] [17].

Another group of electronic circuits made for the high reliability use. This group includes a variety of medical, safety, military technology, aviation and aerospace applications. Reliability of these beyond, are often formulated as special needs, low weight, adaptivity, the reconfigurability, the circuit robustness [6] [4] [7].

In this article we test the different possibilities for such operations the creation of robust circuits, in particular the use of programmable analog circuits.
\end{abstract}

\section{SPECIAL ASPECT OF CIRCUIT ROBUSTNESS}

The reliability of electronic circuits $\left(R_{C}\right)$, the degree of robustness depends on several parameters. So depending on the reliability of the components $\left(R_{p}\right)$, the used manufacturing technology $(M)$, environmental parameters $(E)$, and the circuit definition of quality $(Q)$. In short form according equation (1) is;

$$
R_{C}=\mathfrak{f}\left(R_{p}, M, E, Q\right)
$$

Reliability of components are manufacturing data, its value depends on art of electronic part (resistor, semiconductor, capacitor,..,etc.), and their type of pins (outlets). A quality of ready printed board depends of art of mounting technology, and used additives auxiliary materials. Environmental distinction basically means different physical parameters for example temperature, humidity or even fluctuation of power voltage [16] [15] [14].

Not exact definition of circuit robustness is; be operational at changed circumstances. To the corrected description we must give definition of quality of robustness in (2) equation;

$$
Q_{R_{\min }} \leq\left(\left.\frac{P_{\text {out }}}{P_{\text {in }}}\right|_{s \leqslant k}\right)<Q_{R_{\max }}
$$

where; $Q_{R_{\min }}$, and $Q_{R_{\max }}$ are the parameters of of the specified transfer function of electronic circuit, $s$ is a relevant environmental parameter, $k$ is a prognosticated limit of $s$.

The quality parameter of course other characteristics can also define according equation (2), up to temperature, the maximum power consumption, distortion and so on [8] [9] [5].

\section{SEnSING IN ELECTRONIC CIRCUIT}

General structure a complicated multi-grade circuit can be observed in Fig. 1. The figure's analog circuit built on the subsystems $\left(F_{1}, F_{2}, F_{3}, \ldots, F_{7}\right)$ in which to consider analog electrical parameters $\left(\gamma_{1}, \gamma_{2}, \gamma_{3}, \ldots, \gamma_{7}\right)$. They selected an analog multiplexer $\left(a_{M U X}\right)$ using. The multiplexer switches to it's output $(\gamma)$ the attributes of analog inputs, according binary value of select input $(S)$. This is the traditional polling method which is slows an embedded microcontroller operation. In such cases, an analog-to-digital conversion is needed as well.

If the multiplexer circuit $\left(a_{M U X}\right)$ of Fig. 1 space a digital multiplexer circuit is used, to be prepared in digital sensed signal.

To detect of different electrical characteristics traditional multiple solution exist. In the Fig. 2 an implementation the current sensing is shown. The transistor circuit is to detect the current of collector resistor $\left(R_{L}\right)$, which is a load. As usual solution to the transistor emitter a resistor $\left(R_{S}\right)$ insert, and write: $I_{R L} \simeq I_{R L}^{\prime}$. Si the function of comparator $\left(S_{t}\right)$ is seen in equation (3):

$$
U_{D_{S}}=\left\{\begin{array}{lll}
H & \text { if } & R_{S} \cdot I_{R L}^{\prime} \geq U_{S_{t}}+\frac{h}{2} \\
L & \text { if } & R_{S} \cdot I_{R L}^{\prime}<U_{S_{t}}-\frac{h}{2}
\end{array}\right.
$$

where; $U_{S_{t}}$ threshold voltage of comparator, $h$ is hysteresis of comparator.

In the Fig. 3 we can detect voltage signal $\left(U_{R L}\right)$ on a load $\left(R_{L}\right)$. In the P channel FET drain circuit the load resistor's $\left(R_{L}\right)$ voltage greater the comparator threshold level, then generates $\left(U_{D_{S}}\right)$ output, according shows the equation (3).

We are able to create such a circuit part, by there from any relevant electrical parameter can generate the specifying digital output [3].

\section{Digital INTERVENTION IN TO ELECTRONIC CIRCUIT}

The introduction described on follows that a circuit can significant impact the robustness if the circuit has some reconfiguration capability. 


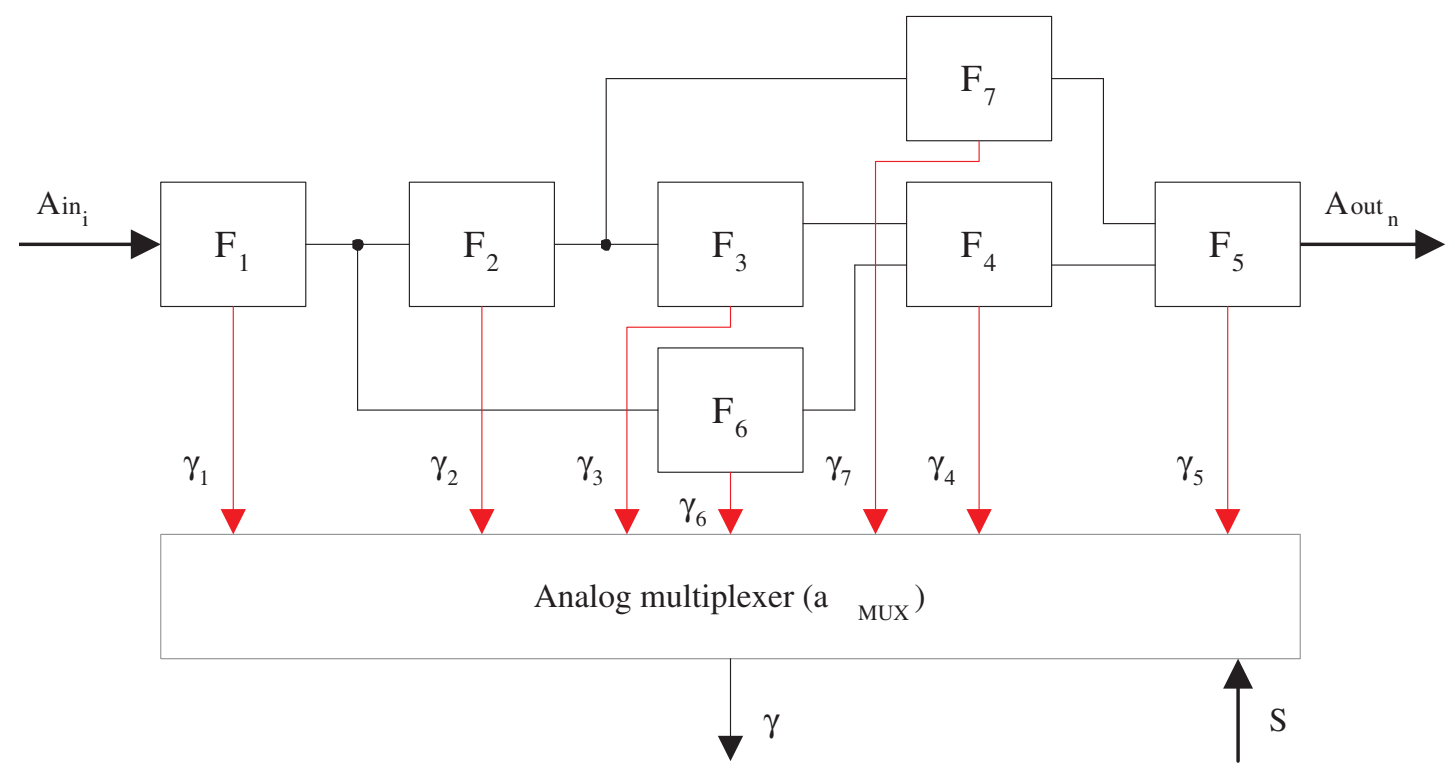

Fig. 1. Theoretical structure of a multistage analog electronic circuit with an analog sensing.

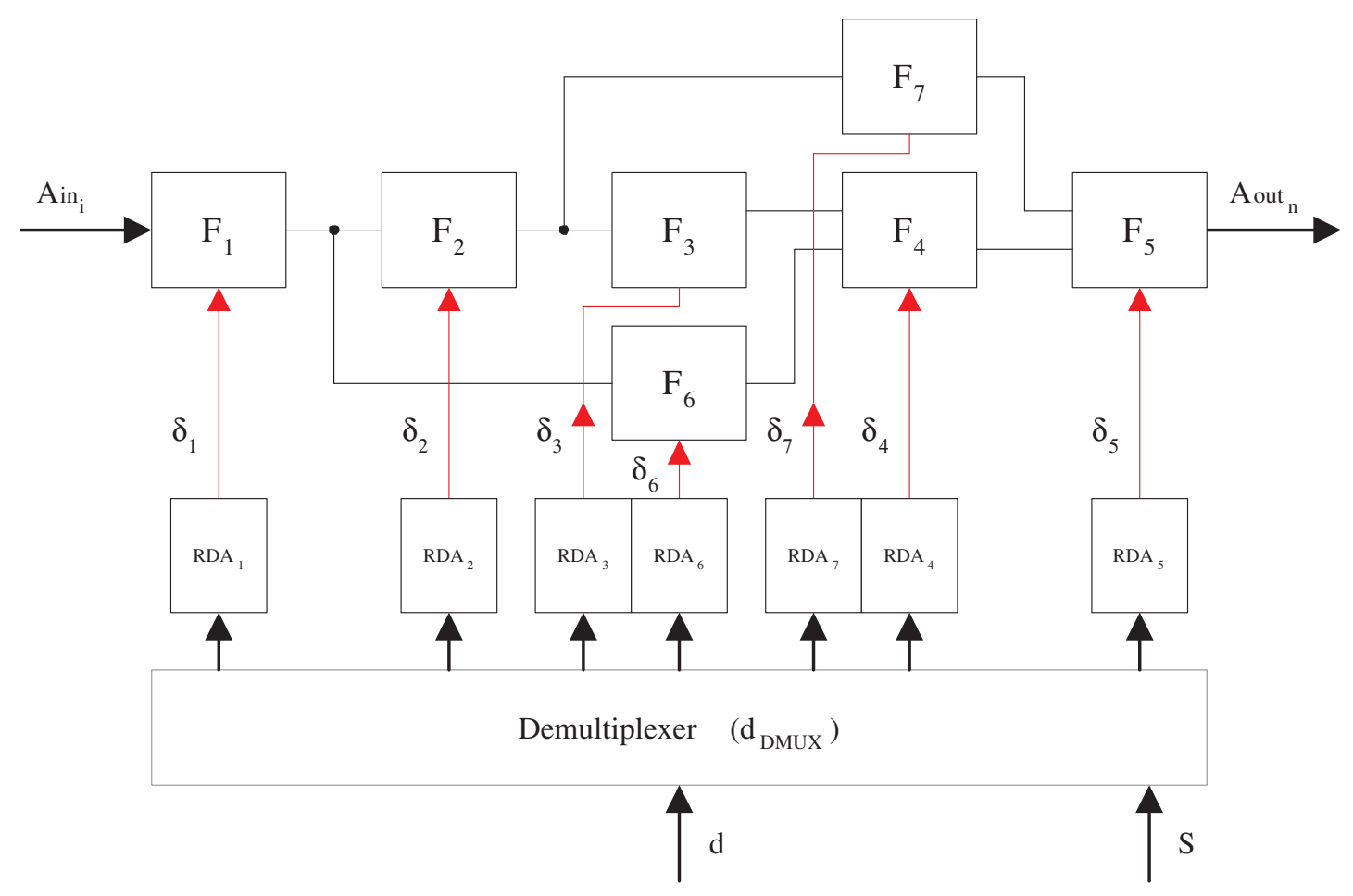

Fig. 4. Digital interaction with RDA modules to sub circuits of an analog circuit system. 


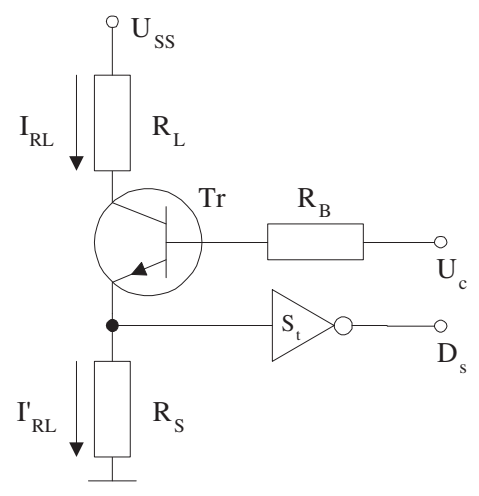

Fig. 2. Current $\left(I_{R L}\right)$ sensing in a BJT amplifier circuit.

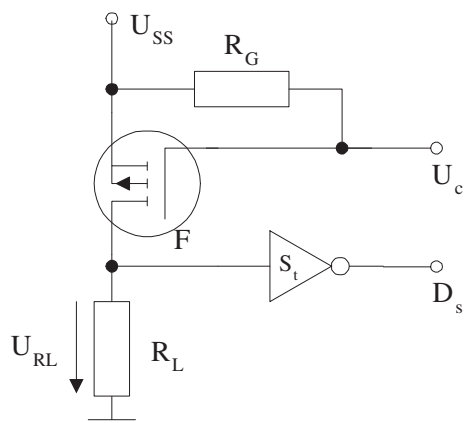

Fig. 3. Voltage $\left(U_{R L}\right)$ sensing in FET amplifier circuit.

As shown in Fig. 4, whole analog circuit consist of the elementary circuits $\left(F_{1}, F_{2}, F_{3}, \ldots, F_{7}\right)$. Relevant parameters of these elementary circuits can modify by the $\sigma_{1}, \sigma_{2}, \sigma_{3}, \ldots, \sigma_{7}$ signals, according the selection of a digital de-multiplexer $\left(d_{D M U X}\right)$ circuit.

To make such intervening by digital signals to analogue characteristics, a RAM-DAC-Amplifier (RDA) unit interposed into the system. Structure of RDA unit used in such cases are shown in Fig. 5.

This unit consists of a RAM, a digital-analog converter and an amplifier. The RAM contains such digital information, which is after digitization and amplification carried out of relevant analog characteristics changes. The Fig. 5 shows a circuit detail by there able to change capacity. DC voltage appearing on the output of the amplifier changes the capacitance of the varicap-diode $\left(D_{v}\right)$ through $R_{v}$ resistor which modifies the ground point relative capacity $\left(C_{v}\right)$ with using a coupling capacitor $\left(C_{a}\right)$.

Such interventions can be observed in Fig. 6. In this case

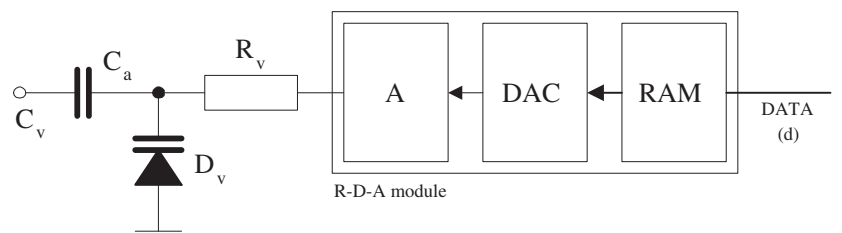

Fig. 5. Structure of RDA actuator circuit in a capacitor modifying application.

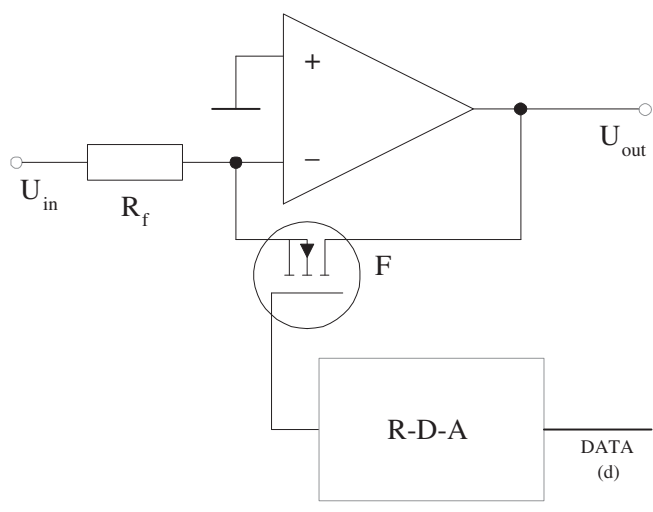

Fig. 6. Amplifying correction by using of RDA unit.

the gain of inverting amplifier is modified by changing the feedback resistance value.

Here, the $R_{D S}$ resistance depends on the $U_{G S}$ voltage at FET $(F)$, so the amplifying factor of operation amplifier depends on the feedback impedance. So in short we can write in equation (4):

$$
\frac{U_{\text {out }}}{U_{\text {in }}}=\frac{R_{D S}}{R_{f}},
$$

were; $R_{D S}$ drain-source resistance of FET its value depends of RDA voltage output, so actually the binary content of RAM.

\section{ANALOG INTERVENTION IN TO ELECTRONIC CIRCUIT}

Fig. 7 shows an analog type of intervention circuit schematic. In the circuit, the $\sigma_{n}$ input signal switched to a sampling module $(S-H)$ according the value of $s_{n}$ sample signal. The storage capacity $\left(C_{S}\right)$ of the operational amplifier $(A)$ feedback branch located in the current voltage charging, and after opening the electronic switch retains this value. Example of the output voltage of $S-H$ unit changes the frequency of an voltage controlled oscillator $(V C O)$.

This type of analog intervening circuit can be in system organized, how mentioned Fig. 8 .

In this case, an analog de-multiplexer $\left(a_{D M U X}\right)$ circuit is used, the input of which is coupled to an analog signal. The analog input signal $(I)$ is a function of the select input $(S)$ will actual value of certain $S_{1}, S_{2}, S_{3}, \ldots, S_{7}$ outputs. These outputs connects to sample and hold circuit which belonging to each analog part circuit. The sample and hold circuits, the

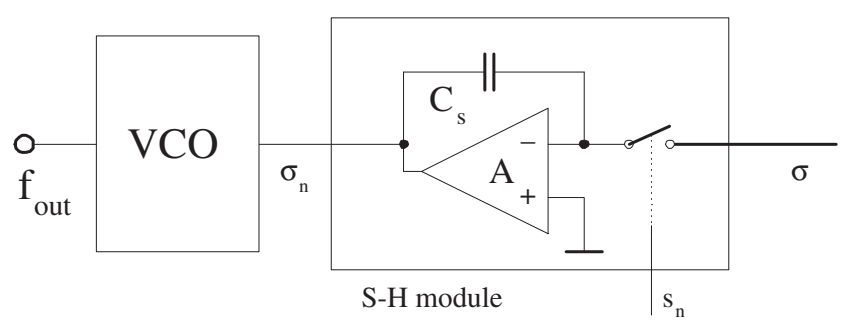

Fig. 7. A circuit which enables analog intervention-signals. 


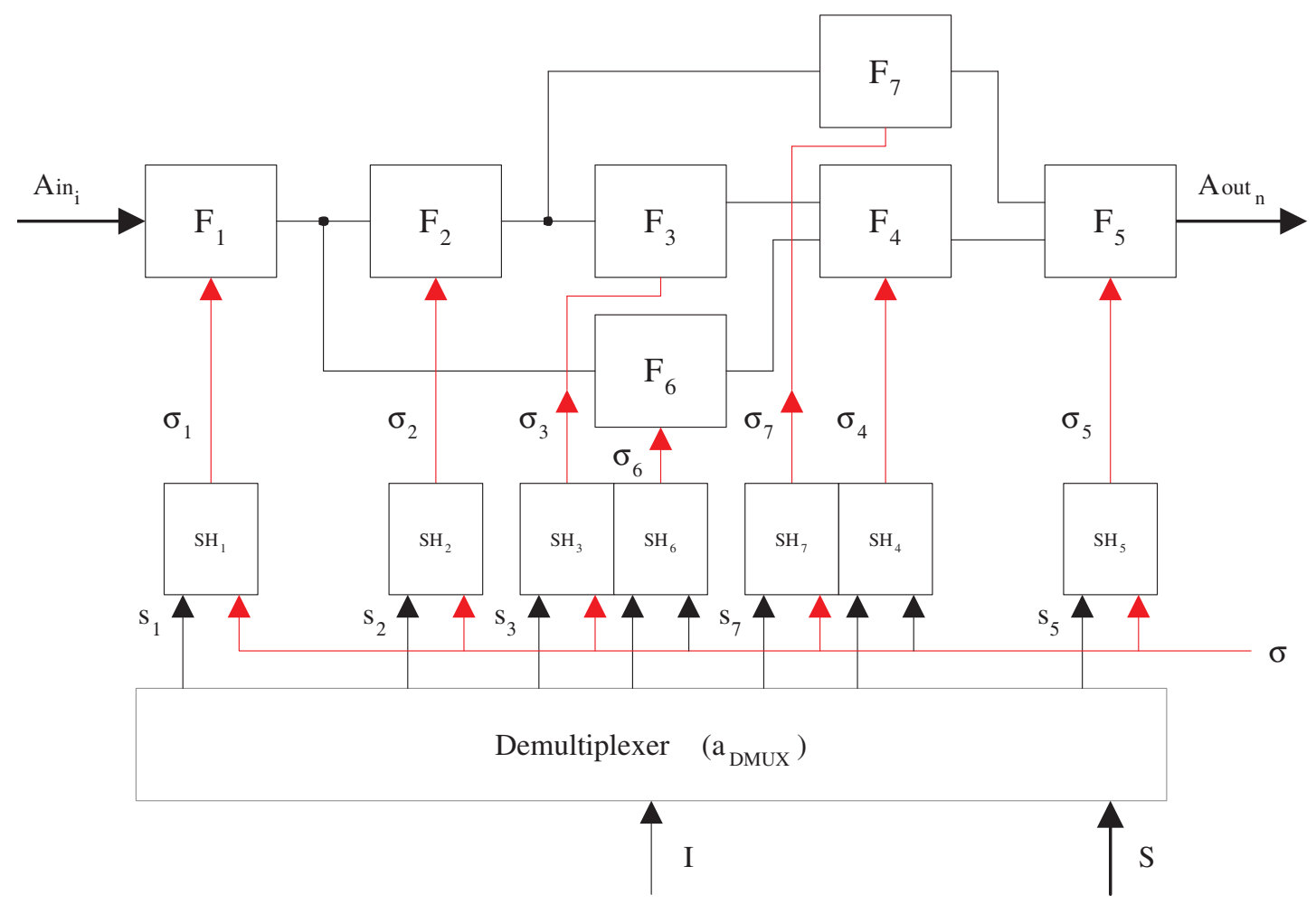

Fig. 8. Analog control signal sharing with an analog demultiplexer and store with sample and hold circuits.

control function $(\sigma)$ of the sample and held, and thereafter the operation of the analog circuit modules to be altered by the current analog value.

\section{A SPECIAL ABILITIES OF USING OF FPAA}

New opportunity is a solution where a polling of a reconfigurable circuit can carry out, be continuously reconfiguration. This is an excellent opportunity for the Field Programmable Analog Arrays (FPAA). In the current query point can be determined by changing the internal structure of the circuit. In this way, a latent analog multiplexer is provided, which addressed depending on the configuration of the lead point, being the desired value of the analog output of circuit.

In Fig. 9, 10, 11 are demonstrated the pragmatical application of the described virtual multiplexer $\left(v_{M U X}\right)$. Basically, a special synthesis filter circuitry in the FPGA. Structures of these do not change [11] [10].

To $07, O 8$ digital output connect ( $n_{10}$ inner node)to a SAR type analog-digital converter. The $n_{8}$ input of DAC connect to output of a sample-and-hold circuit $\left(Z^{-1}\right)$.

In Fig. 9 easy follow that the input of sample-and-hold circuit connect to $n 3$, in Fig. 10 connect to $n 4$, and to $n 9$ in Fig. 11.

Formally described in equation (5):

$$
D_{78}=\mathfrak{g}\left(t_{Z^{-1}}, k_{A D C}, U_{n}, \omega_{n}\right),
$$

were; $D_{78}$ value of FPAA's configured digital output, $t_{Z^{-1}}$ time of sampling, $k_{A D C}$ is the transfer function oaf analog-digital converter, and $\omega_{n}$ is the defined measuring, observed point of FPAA, depends on loaded, reloaded configuration.

This principle can be used to directly drive the analog signal to FPAA's output. In this case, there is no need for the sample and hold circuit, or the analog-digital converter. [19] [2]

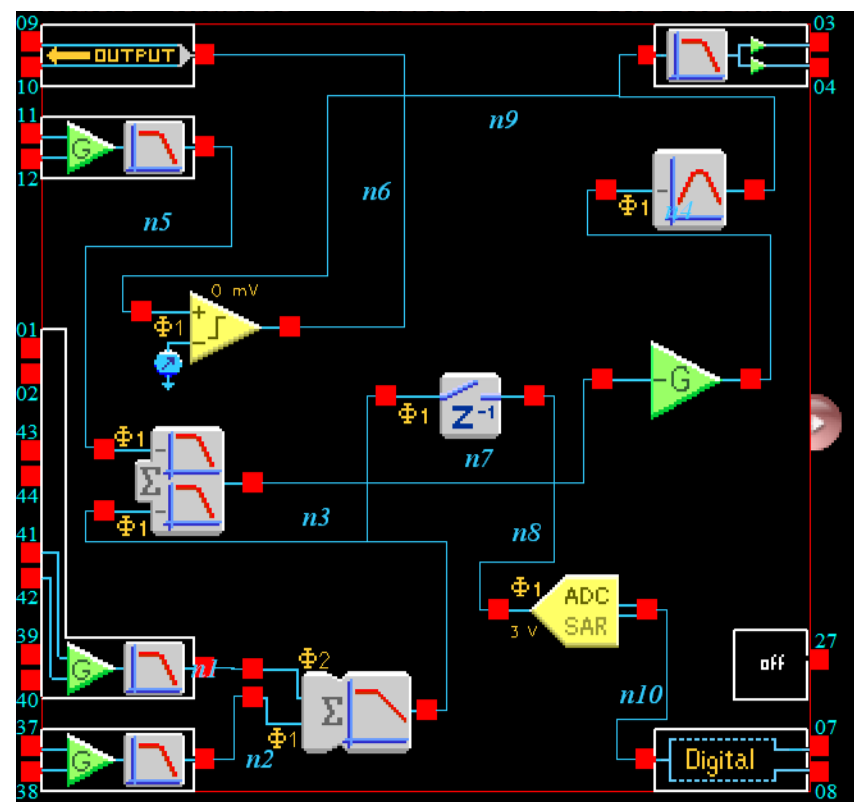

Fig. 9. Virtual multiplexer in FPAA; $\Phi_{1}=n_{3}$. 


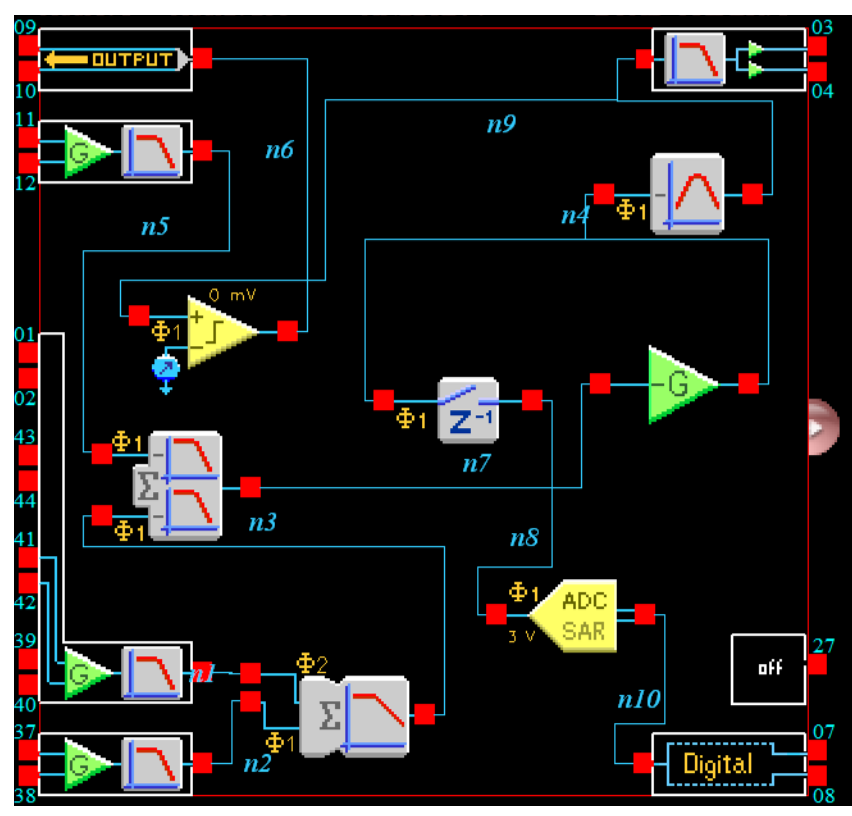

Fig. 10. Virtual multiplexer in FPAA; $\Phi_{1}=n_{4}$.

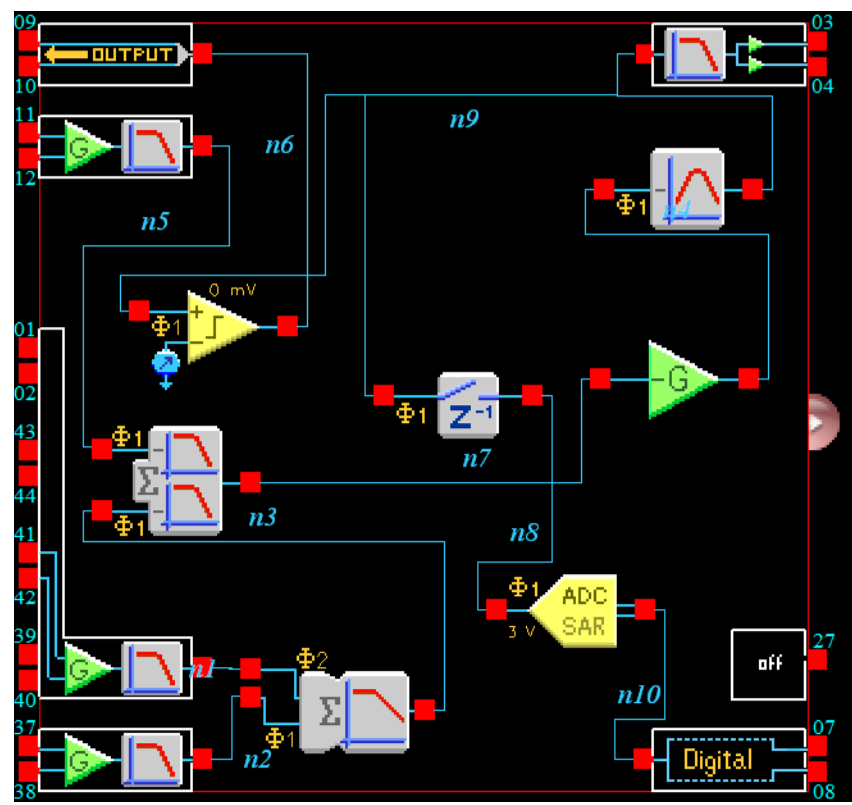

Fig. 11. Virtual multiplexer in FPAA; $\Phi_{1}=n_{9}$.

\section{ROBUST CIRCUIT SYSTEMS}

In above chapters it can be seen that the degree of a circuit robustness can be significantly increased if the circuit can be controlled in some way, if the circuit can be made adaptive. This requires an intervening processes has been shown as examples, there are generally used, and a kind of procedure can be made usable (Fig. 5-7). We measured the change of characteristics of the relevant circuit-part, a method is employed which interrogates the characteristics of analog multiplexer, or digitizing the monitored parameters, and a digital multiplexer to do is retrieve (Fig. 1, 4).
In the case of procedure analog signals, analog values stored in the special circuit is needed (Fig. 8).

\section{CONCLUSIONS}

Programmable analog circuits (FPAA), it is possible to implement a so-called virtual multiplexer feature that other functional units of the circuit is unchanged irrespective of different points of value queries. This is basically an analog or digital means versa.

If you wish to process an analog signal value, a driver amplifier interposing the voltage of selected point you want to retrieve an output delivered. The further processing of voltage of decide ominous point, we can suspect that the voltage is in the correct range.

In another proposed method, the voltage of examined point is sampled and digitized. Thus, a digital signal on the output of the programmable analog circuit microcontroller direct cooperation to handle.

\section{REFERENCES}

[1] N. Ádám. Single input operators of the df kpi system. Acta Polytechnica Hungarica, 7(1):73-86, 2010.

[2] A.S. Deese, C.O. Nwankpa, J. Jimenez, J. Berardino, and J. Hill. Design of modular field programmable analog array hardware for analysis of large power systems. pages 1207-1210, 2012.

[3] Gy. Györök, M. Makó. Configuration of EEG input-unit by electric circuit evolution. pages 1-7, September 2005.

[4] Gy. Györök, M. Makó, J. Lakner. Combinatorics at electronic circuit realization in FPAA. Acta Polytechnica Hungarica, Journal of Applied Sciences, 6(1):151-160, 2009.

[5] Gy. Györök. The function-controlled input for the IN CIRCUIT equipment. pages 443-446, September 2006.

[6] Gy. Györök. Self configuration analog circuit by FPAA. pages 34-37, January 2006.

[7] Gy. Györök. Self organizing analogue circuit by monte carlo method. pages 34-37, September 2007.

[8] Gy. Györök. A-class amplifier with FPAA as a predictive supply voltage control. pages 361-368, November 2008.

[9] Gy. Györök. The FPAA realization of analog robust electronic circuit. pages 1-5, November 2009.

[10] A. Pilat and J. Klocek. Programmable analog hard real-time controller [programowalny sterownik analogowy]. Przeglad Elektrotechniczny, 89(3 A):38-46, 2013. cited By (since 1996) 0.

[11] Adam Pilat. Control toolbox for industrial programmable analog controllerembedding state feedback controller. pages 1-4, 2012.

[12] S. Sergyán. Edge detection techniques of thermal images. 2012 IEEE 10th Jubilee International Symposium on Intelligent Systems and Informatics, SISY 2012, pages 227-231, 2012.

[13] S. Sergyán. Useful and effective feature descriptors in content-based image retrieval of thermal images. LINDI 2012 - 4th IEEE International Symposium on Logistics and Industrial Informatics, Proceedings, pages 55-58, 2012.

[14] J. Tick. User interface redesign based on user behavior analyses. pages 29-31, October 2003

[15] J. Tick. Potential Application of P-Graph-Based Workflow in Logistics. Aspects of Computational Intelligence: Theory and Applications: Revised and Selected Papers of the 15th IEEE International Conference on Intelligent Engineering Systems 2011, pp. 293-303, Springer Verlag, 2012, Heidelberg; London; New York, 2012.

[16] J. Tick. Business process-based initial modeling at software development. pages 141-144, January 2013

[17] Z. Vámossy. Thermal image fusion. 2012 IEEE 10th Jubilee International Symposium on Intelligent Systems and Informatics, SISY 2012, pages 385-388, 2012.

[18] L. Vokorokos, N. Ádám, and B. Madol. The process control for psingle operators. 19th International Workshop on Robotics in AlpeAdria-Danube Region, RAAD 2010 - Proceedings, pages 119-123, 2010. 
[19] Y. Wang, M. Zhu, J. Cui, H. Lin, and Y. Jiang. Current-mode reconfigurable analog circuit for analog signal processing. Nanjing Hangkong Hangtian Daxue Xuebao/Journal of Nanjing University of Aeronautics and Astronautics, 43(4):532-537, 2011. 